\title{
Effect of adalimumab on work productivity and indirect costs in moderate to severe Crohn's disease: A meta-analysis
}

\author{
David G Binion $\mathrm{MD}^{1}$, Edouard Louis MD $\mathrm{PhD}^{2}$, Bas Oldenburg MD PhD ${ }^{3}$, Parvez Mulani PhD ${ }^{4}$, \\ Arielle G Bensimon ${ }^{5}$, Mei Yang $\mathrm{PhD}^{4}$, Jingdong Chao $\mathrm{PhD}^{4}$
}

\begin{abstract}
DG Binion, E Louis, B Oldenburg, et al. Effect of adalimumab on work productivity and indirect costs in moderate to severe Crohn's disease: A meta-analysis. Can J Gastroenterol 2011;25(9):492-496.
\end{abstract}

OBJECTIVE: To assess the effect of adalimumab on work productivity and indirect costs in patients with Crohn's disease (CD) using a metaanalysis of clinical trials.

METHODS: Study-level results were pooled from all clinical trials of adalimumab for moderate to severe CD in which work productivity outcomes were evaluated. Work Productivity and Activity Impairment Questionnaire outcomes (absenteeism, presenteeism and total work productivity impairment [TWPI]) were extracted from adalimumab trials. Meta-analyses were used to estimate pooled averages and $95 \%$ CIs of one-year accumulated reductions in work productivity impairment with adalimumab. Pooled averages were multiplied by the 2008 United States national average annual salary $(\$ 44,101)$ to estimate per-patient indirect cost savings during the year following adalimumab initiation.

RESULTS: The four included trials (ACCESS, CARE, CHOICE and EXTEND) represented a total of 1202 employed adalimumabtreated patients at baseline. Each study followed patients for a minimum of 20 weeks. Pooled estimates (95\% CIs) of one-year accumulated work productivity improvements were as follows: $-9 \%(-10 \%$ to $-7 \%)$ for absenteeism; $-22 \%(-26 \%$ to $-18 \%)$ for presenteeism; and $-25 \%$ $(-30 \%$ to $-20 \%)$ for TWPI. Reductions in absenteeism and TWPI translated into per-patient indirect cost savings (95\% CI) of $\$ 3,856$ ( $\$ 3,183$ to $\$ 4,529)$ and $\$ 10,964$ ( $\$ 8,833$ to $\$ 13,096)$, respectively.

CONCLUSION: Adalimumab provided clinically meaningful improvements in work productivity among patients with moderate to severe $\mathrm{CD}$, which may translate into substantial indirect cost savings from an employer's perspective.

Key Words: Adalimumab; Crohn's disease; Indirect costs; Work productivity

Crohn's disease (CD) is a chronic inflammatory bowel disease usually diagnosed during the second or third decade of life (1), and can have a debilitating effect on patients' ability to work. Indirect costs of work productivity impairment contribute significantly to the economic burden of CD (2-5). In a large, multicentre clinical trial conducted in North America, Europe and Israel (2), 25\% of patients with moderate to severe $\mathrm{CD}$ reported receiving some form of disability compensation at baseline. A systematic literature review (4) found that indirect costs associated with CD-related work disability accounted for $28 \%$ of the total societal cost of CD in the United States (US) and $64 \%$ to $69 \%$ in Europe.

Analyses of the effects of biological therapies for CD on productivity outcomes are needed given the high indirect cost burden of CD (6). Improvements in work productivity and employment outcomes have

\section{L'effet de l'adalimumab sur la productivité au travail et les coûts indirects en cas de maladie de Crohn modérée à grave : une méta-analyse}

\begin{abstract}
OBJECTIF : Évaluer l'effet de l'adalimumab sur la productivité au travail et les coûts indirects chez les patients atteints de la maladie de Crohn (MC) au moyen d'une méta-analyse d'essais cliniques.

MÉTHODOLOGIE : Les résultats des études proviennent de tous les essais cliniques sur l'adalimumab dans le traitement de la MC modérée à grave et qui en évaluaient les résultats sur la productivité au travail. Les résultats du questionnaire sur la productivité au travail et la détérioration de l'activité (absentéisme, présentéisme et détérioration de la productivité totale au travail [DPTT]) ont été tirés des essais sur l'adalimumab. Les méta-analyses ont permis d'évaluer les moyennes groupées et les $95 \%$ IC quant à la réduction accumulée de la détérioration de la productivité au travail en un an grâce à l'adalimumab. Les moyennes groupées ont été multipliées par le salaire annuel moyen national des États-Unis en 2008 (44 101 \$) pour évaluer les économies sur les coûts indirects par patient pendant l'année suivant l'amorce de l'adalimumab.
\end{abstract}

RÉSULTATS : Les quatre essais inclus (ACCESS, CARE, CHOICE et EXTEND) représentaient un total de 1202 patients employés traités à l'adalimumab en début d'étude. Chaque étude a suivi les patients pendant au moins 20 semaines. Les évaluations groupées (95\% IC) d'améliorations de la productivité au travail accumulées en un an s'établissaient comme suit : $9 \%(-10 \%$ à $-7 \%)$ pour l'absentéisme, $-22 \%(-26 \%$ à $-18 \%)$ pour le présentéisme et $25 \%(-30 \%$ à $-20 \%)$ pour la DPTT. La réduction de l'absentéisme et de la DPTT se traduisai par des économies sur les coûts indirects par patient (95\% IC) de 3856 \$ (3 183 \$ à 4529 \$) et de 10964 \$ (8 833 \$à 13096 \$), respectivement.

CONCLUSION : L'adalimumab assurait des améliorations significatives sur le plan clinique en matière de productivité au travail chez les patients ayant une MC modérée à grave, qui peuvent se traduire par des économies importantes sur les coûts indirects du point de vue de l'employeur.

been reported in patients with moderate to severe $\mathrm{CD}$ who were treated with certolizumab pegol (7). In addition, preliminary data suggest a positive effect of adalimumab treatment on work productivity outcomes (8-11). The objective of the present meta-analytic study of adalimumab clinical trials was to derive a comprehensive estimate of the effect of adalimumab treatment on work productivity in patients with moderate to severe $\mathrm{CD}$ and to estimate the associated one-year indirect cost savings from a societal perspective.

Clinical trials and data collection

Data were extracted from all registered, global clinical trials of adalimumab in adult patients with moderate to severe CD in which outcomes based on the Work Productivity and Activity Impairment

\footnotetext{
${ }^{1}$ Division of Gastroenterology, Hepatology and Nutrition, University of Pittsburgh, Pittsburgh, Pennsylvania, USA; ${ }^{2}$ University of Liège and CHU Liège,

Liège, Belgium; ${ }^{3}$ University Medical Centre, Utrecht, The Netherlands; ${ }^{4}$ Abbott Laboratories, Abbott Park, Illinois; ${ }^{5}$ Analysis Group Inc, Boston,

Massachusetts, USA

Correspondence: Dr David G Binion, Division of Gastroenterology, Hepatology and Nutrition, University of Pittsburgh, PUH, M2,

C Wing, 200 Lothrop Street, Pittsburgh, Pennsylvania 15213, USA. Telephone 412-648-9115, fax 412-648-9378, e-mail binion@pitt.edu Received for publication December 17, 2010. Accepted March 28, 2011
} 
Questionnaire (WPAI) were evaluated. Included trials were also required to feature an adalimumab-treated arm receiving $160 \mathrm{mg} / 80 \mathrm{mg}$ induction at weeks 0 and 2, and 40 mg every-other-week maintenance dosing and to follow patients for a minimum of four weeks.

A systematic search of the www.ClinicalTrials.gov registry up to December 2010 was conducted using the phrases "adalimumab" and "Crohn's disease," as well as a variation of this search using the brand drug name. The review identified 13 completed trials of adalimumab for the treatment of $\mathrm{CD}$. Of these trials, one study evaluating adalimumab therapy among pediatric patients with $\mathrm{CD}$ was excluded (ClinicalTrials.gov identifier: NCT00409682). Also excluded were eight clinical trials and open-label extension studies that did not collect WPAI outcomes (ClinicalTrials.gov identifiers: NCT00055497, NCT00445939, NCT01070303, NCT00445432, NCT00195715, NCT00077779, NCT00105300 and NCT00055523). The four remaining trials were eligible for inclusion in the present meta-analysis. ACCESS (A Multicenter, Open-Label, Treatment Protocol of the Human Anti-TNF Monoclonal Antibody Adalimumab in Canadian SubjeCts With ModErate to Severe Crohn's DiSease; NCT00427921) (9), CARE (Crohn's Patients Treated With Adalimumab: Results of a Safety and Efficacy Study; NCT00409617) (10) and CHOICE (Crohn's Disease WHO Failed Prior Infliximab to Collect Safety Data and Efficacy via Patient-Reported Outcome Measures; NCT00338650) (8) were large, phase IIIb, open-label trials. EXTEND (EXTend the Safety and Efficacy of Adalimumab Through ENDoscopic Healing; NCT00348283) (11) was a phase III, randomized, double-blinded, placebo-controlled trial.

Patients enrolled in the four clinical trials were men and women 18 to 75 years of age with CD confirmed by endoscopic or radiological evaluation. In all trials, eligible patients had moderately to severely active CD (Crohn's Disease Activity Index [CDAI] $\geq 220$ and $\leq 450$ in EXTEND; CDAI $>220$ or Harvey-Bradshaw Index [HBI] $\geq 7$ in ACCESS; HBI $\geq 7$ in CARE; and based on physician assessment in CHOICE) for at least four months before screening. The EXTEND trial also required a confirmed diagnosis of ileocolonic CD within three years before the baseline visit. Patients enrolled in the ACCESS and CARE trials were refractory to optimal conventional therapies such as 5-aminosalicylic acid, corticosteroids and immunosuppressive therapies (azathioprine, 6-mercaptopurine and methotrexate). The CHOICE trial restricted enrollment to patients with failed response to previous treatment with infliximab including primary nonresponders to infliximab and patients who initially responded to infliximab but discontinued treatment due to adverse events or loss of response. All studies permitted concomitant use of corticosteroids or other conventional therapies for $\mathrm{CD}$ provided that patients were receiving stable dosages at baseline. Each of the trials was performed at sites in the US, Canada and/or Europe.

Adalimumab dosing regimens were similar across the trials. The adalimumab-treated cohort in each study was given an induction dose of $160 \mathrm{mg}$ at baseline and $80 \mathrm{mg}$ at week 2, followed by a maintenance dose of $40 \mathrm{mg}$ every other week starting at week 4 . Patients who did not demonstrate a clinical response or who experienced a disease flare could increase their dosage to $40 \mathrm{mg}$ weekly.

Relevant data were abstracted from each study including mean work productivity outcomes and measures of variance at each study visit (8-11); studies followed patients for 20 weeks (CARE), 24 weeks (ACCESS and CHOICE) or 52 weeks (EXTEND). Average baseline characteristics of the adalimumab-treated cohort from each trial were also obtained.

\section{Outcome measures}

Each trial collected patient responses to the WPAI questionnaire, which is a validated, self-administered tool that assesses the impact of disease on patients' ability to work $(12,13)$. Three WPAI component scores were computed for employed patients: absenteeism (percentage of work hours missed), presenteeism (visual analogue scale [scored from 0 to 10] rating of impairment while working) and total work productivity impairment (TWPI) (absenteeism and presenteeism combined). Improvements in absenteeism, presenteeism and TWPI from baseline to the mid-year visit (range: week 20 to week 28, owing to differences in reporting intervals across trials) within each adalimumab-treated cohort were assessed.

\section{Statistical analysis}

Meta-analyses were conducted in Stata (StataCorp LP, USA) using the metan command (14) to derive pooled estimates and 95\% CIs of improvements in absenteeism, presenteeism and TWPI at weeks 4 and 12 , and mid-year study visits. Random-effects models were used when statistical heterogeneity was present (ie, $\mathrm{P}<0.05)$; otherwise, a fixedeffects model was used. The resulting pooled estimates and standard errors of productivity improvements at week 4, week 12 and mid-year study visits were weighted to derive estimates of patients' average productivity improvements during the year following adalimumab initiation, with the assumption that patients experienced a constant rate of improvement between the baseline, week 4, week 12 and mid-year visits, and subsequently maintained the same average improvement from the mid-year visit to week 52 . Average productivity improvement over one year was calculated as the area under the resulting curve divided by 52 (the number of weeks in a year). Numerically, this area is equivalent to the area under the stepwise curve that would result if it was assumed that the patients maintained their week-0 improvement from weeks 0 to 2 , their week-4 improvement from weeks 3 to 8 , their week-12 improvement from weeks 9 to 19 , and their week-26 improvement from weeks 20 to 52 . Accordingly, the weight of each study visit in the calculation of one-year accumulated productivity improvements was 3.8\% (ie, 2/52) for week 0,11.5\% (6/52) for week 4, $21.2 \%(11 / 52)$ for week 12 and $63.5 \%$ (33/52) for week 26.

Changes in WPAI scores were interpreted based on a minimum clinically important difference of 7\% (15). Pooled absenteeism and TWPI improvements were multiplied by the 2008 US national average annual salary $(\$ 44,101)(16)$ to estimate per-patient one-year indirect cost savings associated with adalimumab.

\section{RESULTS}

Clinical trial results pooled from ACCESS, CARE, CHOICE and EXTEND represented a total of 1202 employed adalimumab-treated patients at baseline (Table 1). Mean baseline TWPI scores ranged from $49 \%$ to $71 \%$ across the trials, indicating substantial work productivity impairment at baseline (Table 1). Within the adalimumabtreated cohort from each trial, clinically significant productivity improvements that exceeded the minimum clinically important difference (ie, 7\%) were observed at week 4 and were sustained to the end of the trial period, with the exception of absenteeism at week 4 in ACCESS (six-point improvement) and week 8 in CHOICE (six-point improvement).

Figure 1 summarizes results from the meta-analyses of changes in WPAI outcomes at the week 4 , week 12 and mid-year study visits. Combining the reported outcomes from all adalimumab-treated cohorts, the pooled estimates of improvements in WPAI scores from baseline to the mid-year visit were $-9.20 \%(95 \% \mathrm{CI}-11.49 \%$ to $-6.91 \%$ ) for absenteeism, $-23.90 \%$ (95\% CI $-29.76 \%$ to $-18.05 \%)$ for presenteeism, and $-26.64 \%$ (95\% CI $-33.73 \%$ to $-19.56 \%$ ) for TWPI. Pooled estimates of productivity improvements at week 4 and week 12 were similar in magnitude. Statistical heterogeneity among the included studies was present in meta-analyses of presenteeism and TWPI ( $P<0.05$ at all study visits) but was not found with respect to absenteeism ( $P>0.20$ at all study visits).

Meta-analysis pooled estimates at individual study visits were aggregated into overall estimates of average improvements in productivity scores over one year. Using weighted averages of pooled estimates at week 4, week 12 and mid-year visits, estimated one-year improvements were $-8.74 \%$ (95\% CI $-10.27 \%$ to $-7.22 \%)$ for absenteeism, $-22.15 \%$ (95\% CI $-26.12 \%$ to $-18.18 \%$ ) for presenteeism and $-24.86 \%$ (95\% CI $-29.70 \%$ to $-20.03 \%$ ) for TWPI (Table 2). 
TABLE 1

Characteristics of adalimumab-treated patient cohorts from studies included in the present meta-analysis

\begin{tabular}{|c|c|c|c|c|c|c|c|c|c|}
\hline \multirow[b]{3}{*}{ Study } & \multirow{3}{*}{$\begin{array}{c}\text { Patients in } \\
\text { adalimumab } \\
\text { cohort, } \mathbf{n}\end{array}$} & \multicolumn{5}{|c|}{ Demographics and disease history* } & \multirow{2}{*}{\multicolumn{3}{|c|}{$\begin{array}{l}\text { Work productivity scores at week } 0^{\dagger} \\
\text { mean } \pm \text { SD }\end{array}$}} \\
\hline & & \multirow{2}{*}{$\begin{array}{l}\text { Employed at } \\
\text { baseline, } \mathbf{n}(\%)\end{array}$} & \multirow{2}{*}{$\begin{array}{l}\text { Age, years, } \\
\text { mean } \pm S D\end{array}$} & \multirow{2}{*}{$\begin{array}{l}\text { Female, } \\
\text { n (\%) }\end{array}$} & \multirow{2}{*}{$\begin{array}{c}\text { Crohn's disease } \\
\text { duration, years, } \\
\text { mean } \pm \text { SD }\end{array}$} & \multirow{2}{*}{$\begin{array}{c}\text { Previous } \\
\text { anti-TNF } \\
\text { therapy, } \mathrm{n}(\%)\end{array}$} & & & \\
\hline & & & & & & & Absenteeism & Presenteeism & TWPI \\
\hline ACCESS & 304 & $195(64.1)$ & $37 \pm 12$ & $173(56.9)$ & $12 \pm 10$ & $144(59.0)$ & $16 \pm 27$ & $50 \pm 25$ & $57 \pm 27$ \\
\hline CARE & 945 & $543(57.5)$ & $35 \pm 11$ & $568(60.1)$ & $10 \pm 8$ & $461(48.8)$ & $23 \pm 34$ & $45 \pm 27$ & $51 \pm 29$ \\
\hline CHOICE & 673 & $423(62.9)$ & $41 \pm 13$ & $398(59.1)$ & $14 \pm 10$ & $673(100.0)$ & $15 \pm 25$ & $44 \pm 27$ & $49 \pm 29$ \\
\hline EXTEND & 64 & $41(64.1)$ & $37 \pm 12$ & $81(62.8)$ & $10 \pm 8$ & $78(60.5)$ & $36 \pm 36$ & $58 \pm 24$ & $71 \pm 24$ \\
\hline
\end{tabular}

${ }^{*}$ Demographics and disease history are described for the full adalimumab cohort from each trial; ${ }^{\dagger}$ Work productivity scores at week 0 were determined for the subset of patients in the adalimumab-treated cohort who were employed at baseline. ACCESS A Multicenter, Open-Label, Treatment Protocol of the Human Anti-TNF Monoclonal Antibody Adalimumab in Canadian SubjeCts With ModErate to Severe Crohn's DiSease; CARE Crohn's Patients Treated With Adalimumab: Results of a Safety and Efficacy Study; CHOICE Crohn's Disease WHO Failed Prior Infliximab to Collect Safety Data and Efficacy via Patient-Reported Outcome Measures; EXTEND EXTend the Safety and Efficacy of Adalimumab Through ENDoscopic Healing; TNF Tumour necrosis factor; TWPI Total work productivity impairment
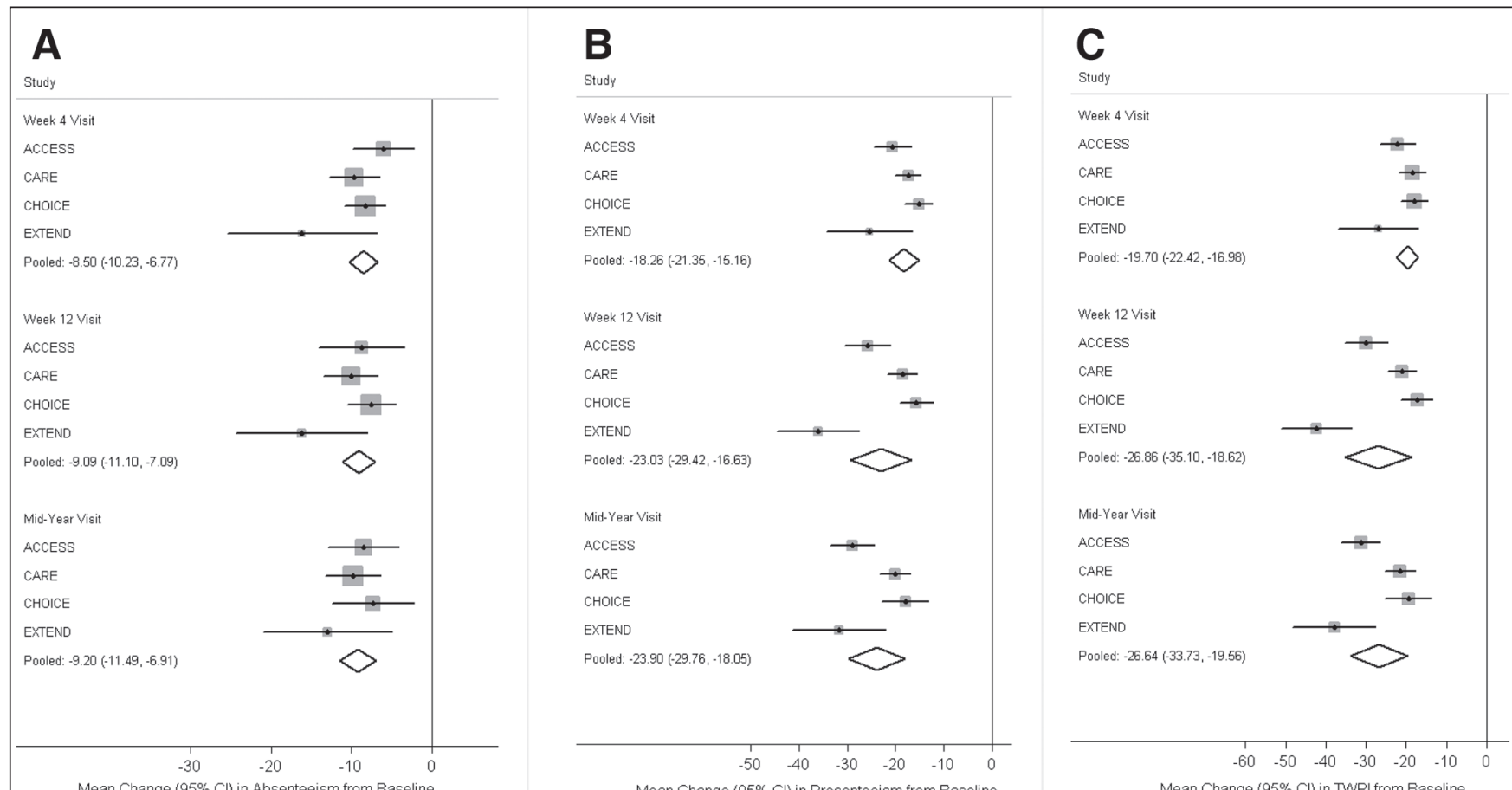

Mean Change $(95 \% \mathrm{Cl})$ in Presenteeism from Baseline Mean Change $(95 \% \mathrm{Cl})$ in TWPI from Baseline

Figure 1) Meta-analysis pooled estimates of work productivity and activity improvements with adalimumab at week 4, week 12 and mid-year study visits. A Absenteeism. B Presenteeism. C Total work productivity impairment (TWPI). The CI for each study is represented by a horizontal line and the point estimate is represented by the centre of a square. The size of the square corresponds to the weight of the study in the meta-analysis. The CIs for pooled estimates are represented by a diamond shape. ACCESS A Multicenter, Open-Label, Treatment Protocol of the Human Anti-TNF Monoclonal Antibody Adalimumab in Canadian SubjeCts With ModErate to Severe Crohn's DiSease; CARE Crohn's Patients Treated With Adalimumab: Results of a Safety and Efficacy Study; CHOICE Crohn's Disease WHO Failed Prior Infliximab to Collect Safety Data and Efficacy via Patient-Reported Outcome Measures; EXTEND EXTend the Safety and Efficacy of Adalimumab Through ENDoscopic Healing

TABLE 2

Average work productivity improvements over one year following initiation of adalimumab therapy

\begin{tabular}{|c|c|c|c|c|}
\hline \multirow[b]{2}{*}{ Study visit } & \multirow{2}{*}{$\begin{array}{c}\text { Weight*, } \\
\%\end{array}$} & \multicolumn{3}{|c|}{ Pooled average productivity improvement according to study visit, mean change, \% ( $95 \% \mathrm{Cl})$} \\
\hline & & Absenteeism & Presenteeism & TWPI \\
\hline Week 0 (baseline) & 3.8 & 0 & 0 & 0 \\
\hline Week 4 & 11.5 & $-8.50(-10.23$ to -6.77$)$ & $-18.26(-21.35$ to -15.16$)$ & $-19.70(-22.42$ to -16.98$)$ \\
\hline Week 12 & 21.2 & $-9.09(-11.10$ to -7.09$)$ & $-23.03(-29.42$ to -16.63$)$ & $-26.86(-35.10$ to -18.62$)$ \\
\hline Week 26 (mid-year) & 63.5 & $-9.20(-11.49$ to -6.91$)$ & $-23.90(-29.76$ to -18.05$)$ & $-26.64(-33.73$ to -19.56$)$ \\
\hline Weighted average improvement over 1 year & - & $-8.74(-10.27$ to -7.22$)$ & $-22.15(-26.12$ to -18.18$)$ & $-24.86(-29.70$ to -20.03$)$ \\
\hline
\end{tabular}

${ }^{*}$ The estimate and $95 \% \mathrm{Cl}$ of average accumulated improvement over the one-year period after adalimumab initiation were derived based on a weighted average of the meta-analysis pooled estimates and standard errors of improvements at the week 4, week 12 and mid-year study visits. The weighting scheme was based on the assumption that patients experienced a constant rate of improvement from baseline to week 4, from week 4 to week 12 , and from week 12 to the mid-year visit, and subsequently maintained the same average improvement from mid-year until week 52. TWPI Total work productivity impairment 
Assuming an average annual salary of $\$ 44,101$, TWPI improvements translated into an estimated indirect costs savings of $\$ 10,964$ (95\% CI $\$ 8,833$ to $\$ 13,096)$ per employed patient over the year following adalimumab initiation, owing to reductions in CD-related work loss and productivity impairment. Pooled results for absenteeism indicated expected cost savings of $\$ 3,856$ (95\% CI $\$ 3,183$ to $\$ 4,529)$ from missed work days alone. The one-year per-patient cost savings from improved productivity while working (presenteeism) was approximated by the difference between TWPI-related and absenteeismrelated indirect cost savings $(\$ 7,108)$.

\section{DISCUSSION}

The present meta-analysis used reported outcomes from four phase III and phase IIIb trials to comprehensively evaluate the work productivity benefits of adalimumab maintenance therapy in patients with moderate to severe $\mathrm{CD}$ and to quantify the associated indirect cost savings per employed patient from an employer payer's perspective.

One strength of the current study was the inclusion of outcomes based on the WPAI instrument at multiple time points, which enabled the estimation of changes in both absenteeism- and presenteeismrelated productivity impairment with therapy over time. Studies included in the meta-analysis were homogeneous in their findings of clinically meaningful improvements from baseline in all components of the WPAI among employed adalimumab-treated patients. These improvements in productivity were rapid and sustained throughout the follow-up period in each trial. Based on meta-analysis results, we estimated that average improvements in work productivity outcomes during the year following adalimumab initiation were $-8.74 \%$ for absenteeism, $-22.15 \%$ for presenteeism and $-24.86 \%$ for TWPI.

The indirect cost burden of CD takes the form of both greater missed work time and reduced productivity while working. Using national average salary data, estimated reductions in work productivity impairment following adalimumab initiation translated into a one-year per-patient cost savings of $\$ 10,964$ owing to decreases in absenteeism and presenteeism. Estimated savings from reduced absenteeism alone was $\$ 3,856$, suggesting that productivity studies that focus only on missed work time are likely to underestimate the impact of CD and its treatment on disease-related work disability.

Previous studies have characterized the high indirect cost burden of $\mathrm{CD}$ and established the correlation between disease activity and both direct medical expenditures and indirect work loss costs due to CD (2-5). However, few studies have assessed the impact of therapy on work productivity outcomes. Results from our meta-analysis of WPAI outcomes with adalimumab are consistent with a recent report of significant reductions in absenteeism $(-12 \%)$, presenteeism $(-18 \%)$ and TWPI $(-21 \%)$ associated with 26 weeks of treatment with certolizumab pegol (7). Additional research is needed to examine the productivity benefits of other biological and conventional therapies for CD to better differentiate treatment options in terms of improving occupational functioning and reducing the indirect cost burden of disease.

Limitations of the present study include heterogeneity across the included trials. For purposes of inclusiveness, the meta-analysis pooled data from all completed registered trials of adalimumab in moderate to severe CD with available WPAI outcomes. Although the trials being combined generally used similar study designs, adalimumab dosing regimens and reporting intervals, differences in patient inclusion criteria may help to explain the cross-trial variations in results. For example, the CHOICE trial required enrolled patients to have previously experienced treatment failure with infliximab. Including this potentially more-difficult-to-treat patient sample in the meta-analysis was a likely source of heterogeneity, and may have resulted in more conservative estimates of the effect of adalimumab on work productivity. Many patients in the ACCESS, CARE and EXTEND trials also experienced nonresponse to previous infliximab therapy. The mix of antitumour necrosis factor-naive patients and infliximab nonresponders across the included trials probably reflects a real-world representation of patients with moderate to severe CD.
Furthermore, although the included trials each followed patients for a minimum of 20 weeks, changes in WPAI scores at one year were evaluated only in EXTEND. Owing to the lack of WPAI data at the one-year end point in the other three trials, the one-year cost model assumed that patients maintained the same average WPAI improvements from the mid-year visit until year end. This assumption was supported by the stability of mean WPAI improvements from week 28 to week 52 in the EXTEND trial (11), and by the sustainability of clinical remission and quality-of-life improvements observed in a previous large-sample, one-year trial of adalimumab $(17,18)$. However, additional studies are needed to establish the maintenance of productivity benefits with biological therapy in the longer term.

\section{CONCLUSION}

Pooled outcomes from four clinical trials demonstrated that adalimumab therapy was associated with significant and clinically meaningful improvements in work productivity for patients with moderate to severe CD. To our knowledge, the present study was the first analysis to show that reductions in work productivity impairment with adalimumab translated into substantial indirect cost savings per employed patient in the year following therapy initiation. The results demonstrate the importance of integrating work productivity outcomes into future economic evaluations of antitumour necrosis factor therapies.

ACKNOWLEDGEMENTS: The authors thank Robin L Stromberg, $\mathrm{PhD}$, of Arbor Communications, Inc, Ann Arbor (Michigan, USA) for medical writing and editing services in the preparation of the manuscript. This support was funded by Abbott.

FINANCIAL SUPPORT: This study was funded by Abbott Laboratories, Abbott Park, Illinois (USA).

DISCLOSURE: Dr Binion has received grants from the National Institutes of Health and UCB Pharma and consulting fees from UCB Pharma. Dr Louis has received research grant support from AstraZeneca and Schering-Plough, speaker fees from Abbott Laboratories, AstraZeneca, Ferring, Schering-Plough and UCB Pharma, and has served as an advisory board member for Abbott Laboratories, Ferring and UCB Pharma. Dr Oldenburg has received grants from Abbott Laboratories, The Netherlands Organisation for Health Research and Development, Schering-Plough, and Tramedico and speaker fees from Ferring Pharmaceuticals. AG Bensimon is an employee of Analysis Group Inc, under contract with Abbott Laboratories for the conduct of this research. Drs Mulani, Yang, and Chao are employees of Abbott Laboratories.

\section{REFERENCES}

1. Lichtenstein GR, Hanauer SB, Sandborn WJ. Management of Crohn's disease in adults. Am J Gastroenterol 2009;104:465-83.

2. Feagan BG, Bala M, Yan S, et al. Unemployment and disability in patients with moderately to severely active Crohn's disease. J Clin Gastroenterol 2005;39:390-5.

3. Stark R, König HH, Leidl R. Costs of inflammatory bowel disease in Germany. Pharmacoeconomics 2006;24:797-814.

4. Yu AP, Cabanilla LA, Wu EQ, et al. The costs of Crohn's disease in the United States and other Western countries: A systematic review. Curr Med Res Opin 2008;24:319-28.

5. Mesterton J, Jönsson L, Almer SH, et al. Resource use and societal costs for Crohn's disease in Sweden. Inflamm Bowel Dis 2009; 15:1882-90.

6. Zisman TL, Cohen RD. Pharmacoeconomics and quality of life of current and emerging biologic therapy for inflammatory bowel disease. Curr Treat Options Gastroenterol 2007;10:185-94.

7. Feagan BG, Reilly MC, Gerlier L, et al. Clinical trial: The effects of certolizumab pegol therapy on work productivity in patients with moderate-to-severe Crohn's disease in the PRECiSE 2 study. Aliment Pharmacol Ther 2010;31:1276-85. 
8. Lichtiger S, Present D, Persson B, et al. T1062 improvement in work productivity with adalimumab in Crohn's disease patients who failed prior infliximab: An analysis of the CHOICE trial. Gastroenterology 2008;134:A475. (Abst)

9. Loftus E, Binion D, Panaccione R, et al. The ACCESS trial: Adalimumab improves work productivity in patients with Crohn's disease. Am J Gastroenterol 2008;103(Suppl 1):S382.

10. Louis E, Lofberg R, Reinisch W, et al. The CARE trial: Adalimumab improves work productivity and quality of life in patients with Crohn's disease (Abst P069). Presented at the Fourth Congress of the European Crohn's and Colitis Organisation. Hamburg, February 5 to 7, 2009.

11. Rutgeerts P, Binion D, Van Assche G, et al. Adalimumab improves work productivity in patients with moderate to severe Crohn's disease: The EXTEND trial (Abst P0333). Presented at Gastro 2009. London, November 21 to 25, 2009.

12. Reilly MC, Gerlier L, Brabant Y, et al. Validity, reliability, and responsiveness of the work productivity and activity impairment questionnaire in Crohn's disease. Clin Ther 2008;30:393-404.

13. Prasad M, Wahlqvist P, Shikiar R, et al. A review of self-report instruments measuring health-related work productivity:
A patient-reported outcomes perspective. Pharmacoeconomics 2004;22:225-44.

14. Egger M, Davey Smith G, Altman DG. Systematic Reviews in Health Care: Meta-analysis in Context, 2nd edn. London: BMJ Publishing Group, 2001.

15. Reilly MC, Brown MCJ, Brabant Y, et al. Minimally important difference for WPAI:CD scores: Defining relevant impact on work productivity in active Crohn's disease (Abst 962). Presented at the American College of Gastroenterology Annual Scientific Meeting. Philadelphia, October 12 to 17, 2007.

16. US Bureau of Labor Statistics. National compensation survey: Occupational earnings in the United States, 2008. <www.bls.gov/ncs/ncswage2008.htm\#Wage_Tables> (Accessed on June 7, 2010).

17. Colombel JF, Sandborn WJ, Rutgeerts P, et al. Adalimumab for maintenance of clinical response and remission in patients with Crohn's disease: The CHARM trial. Gastroenterology 2007;132:52-65.

18. Loftus EV, Feagan BG, Colombel JF, et al. Effects of adalimumab maintenance therapy on health-related quality of life of patients with Crohn's disease: Patient-reported outcomes of the CHARM trial. Am J Gastroenterol 2008;103:3132-41. 


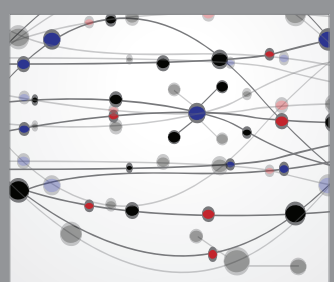

The Scientific World Journal
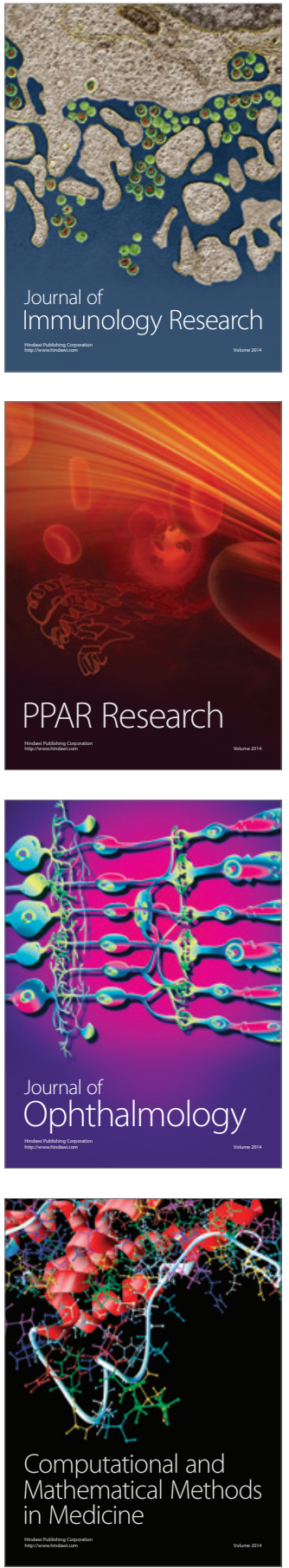

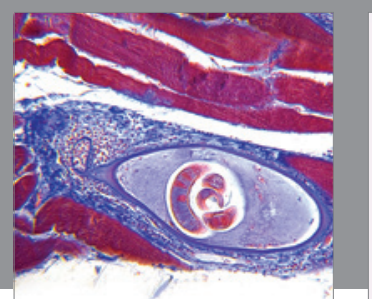

Gastroenterology Research and Practice

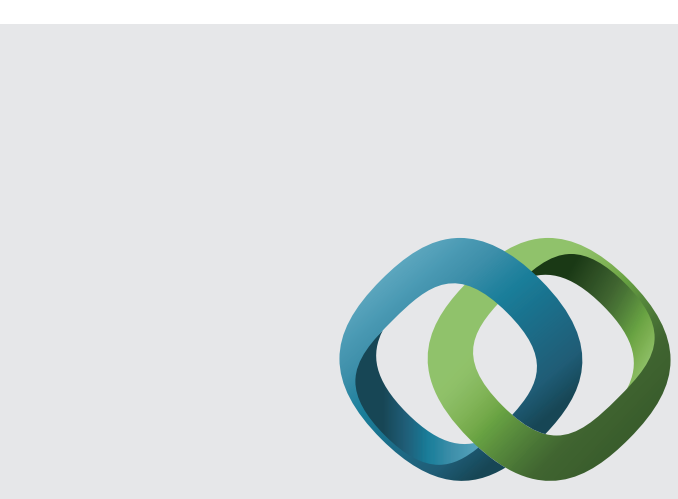

\section{Hindawi}

Submit your manuscripts at

http://www.hindawi.com
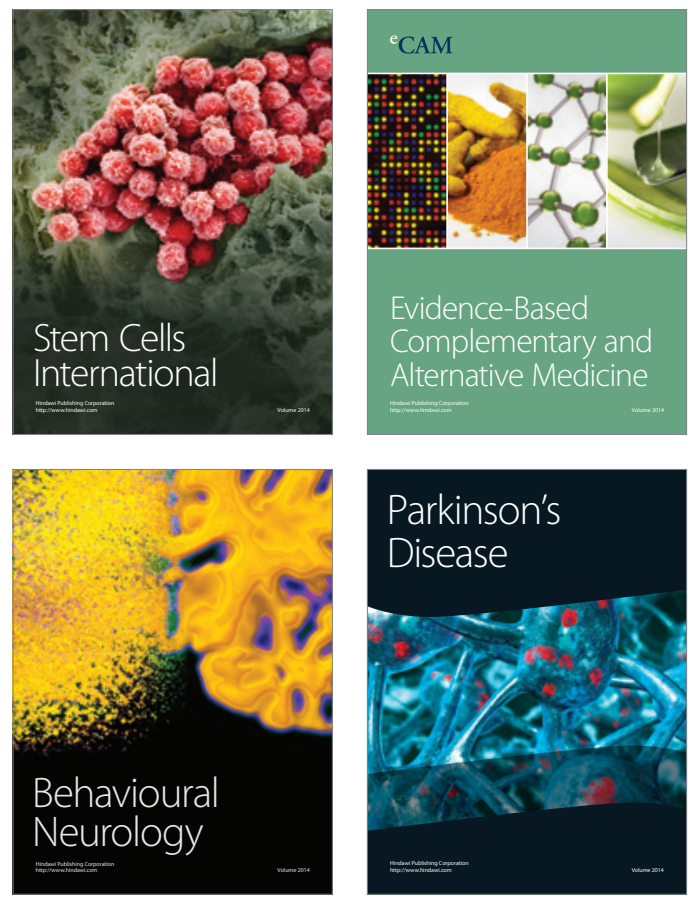
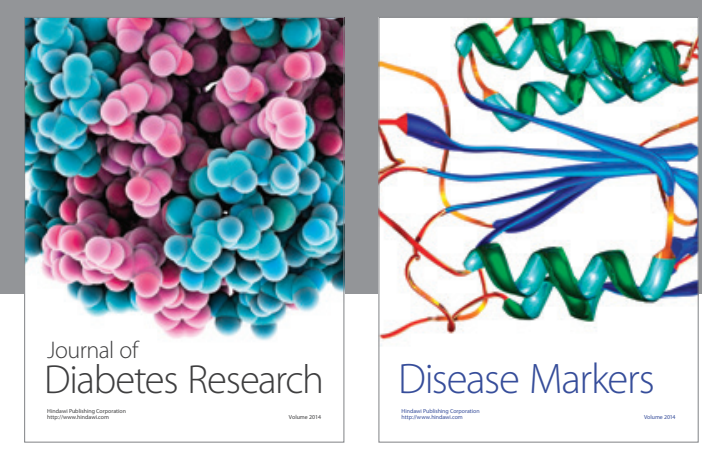

Disease Markers
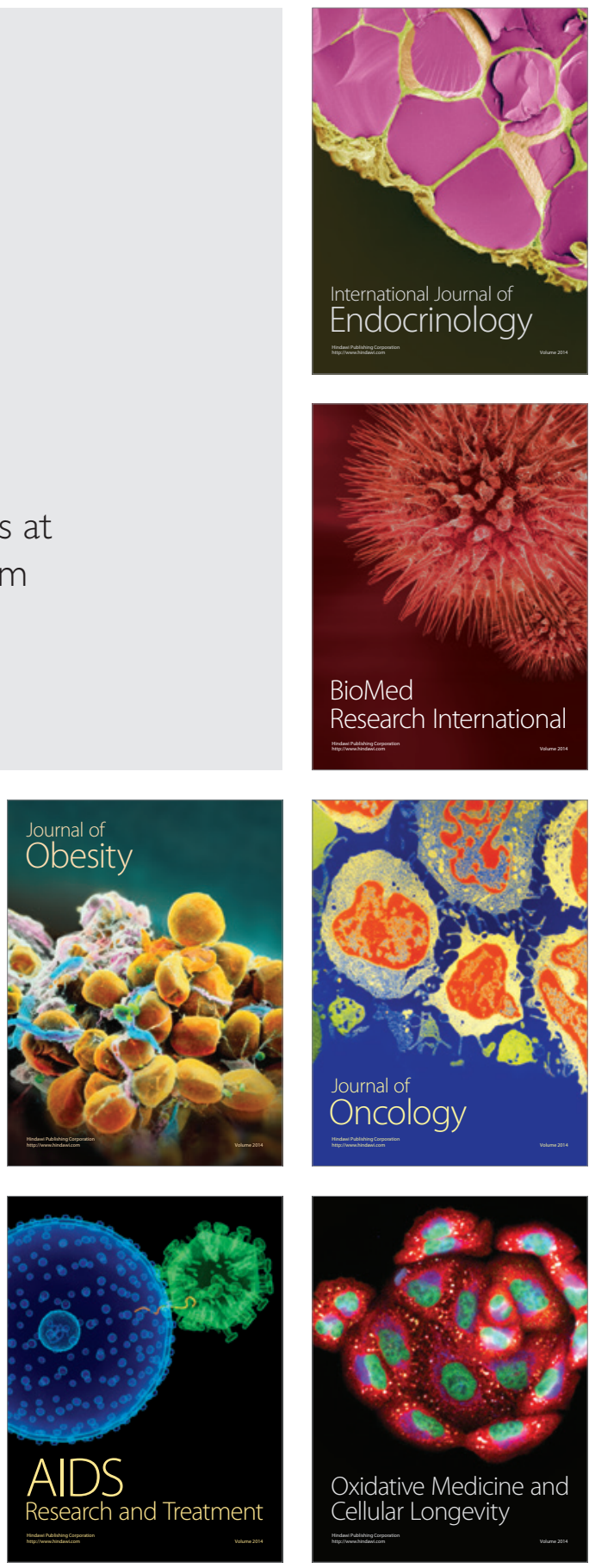